\title{
Outcome following mini-invasive surgical repair of closed Achilles tendon rupture
}

\author{
Omar Al-Fakayh
}

Received: 11 September 2009 /Revised: 12 September 2009/Accepted: 3 November 2009/Published online: 22 November 2009

(C) Springer-Verlag 2009

\section{Dear Editor,}

I read with great interest the study by Mayukh Bhattacharyya and Bruno Gerber in the February 2009 edition and I have a few comments [1]. I would like firstly to thank the authors for the efforts spent to present this study on a very controversial topic in trauma surgery.

It is well known that rupture of Achilles tendon can be treated operatively or non-operatively with equally good long-term results for patients in whom rerupture did not occur [2]. However, operative treatment is preferable in the healthy, active adult group [2, 3].

This paper does raise some queries. Patient selection was done based on the decision of the consultant on call. Also, the first group of 34 patients were allocated for the open method and the second group of 25 patients were allocated for the mini-invasive method. This could create selection bias which might affect the interpretation of the results. I would appreciate the authors' clarification.

One patient from the mini-invasive group with history of HIV was excluded from data analysis because of his absence at the final follow-up. Ideally, the available data of this patient should be analysed with an intention-to-treat approach.

The postoperative rehabilitation was different for both groups. It would be better if the author used a similar rehabilitation programme (e.g. allowing both groups early weight bearing) to support their aim. It has been shown that fully weight bearing after surgical repair will improve functional outcome [4]. Therefore, the mini-invasive group benefited from early weight bearing.
I compliment the authors on adding another facet of information to the natural history of the treatment of these injuries. Also, I would agree with the authors that the comparison between the two groups will give some conclusions about postoperative outcome following a mini-invasive approach. However, the best way of answering this question is to select a group of patients with Achilles tendon ruptures, and then randomise them into two groups to minimise as much as possible the effects of confounding factors. Also, using a single postoperative rehabilitation programme for both groups will help to detect reliable results.

\section{References}

1. Bhattacharyya M, Gerber B (2009) Mini-invasive surgical repair of the Achilles tendon-does it reduce post-operative morbidity? Int Orthop 33(1):151-156

2. Moller M, Movin T, Granhed H, Lind K, Faxen E, Karlsson J (2001) Acute rupture of tendon Achilles. A prospective randomised study of comparison between surgical and nonsurgical treatment. J Bone Jt Surg Br 83(6):843-848

3. Cetti R, Christensen SE, Ejsted R, Jensen NM, Jörgensen U (1993) Operative versus nonoperative treatment of Achilles tendon rupture: a prospective randomized study and review of the literature. Am J Sports Med 21(6):791-799

4. Costa ML, MacMillan K, Halliday D, Chester R, Shepstone L, Robinson AH, Donell ST (2006) Randomised controlled trials of immediate weight-bearing mobilisation for rupture of the tendo Achillis. J Bone Jt Surg Br 88(1):69-77

O. Al-Fakayh $(\bowtie)$

Trauma and Orthopaedics, Aberdeen Royal Infirmary,

Aberdeen, UK

e-mail: alfakayh@hotmail.co.uk 